\title{
Diplorickettsia Bacteria in an Ixodes scapularis Tick, Vermont, USA
}

\section{Carter Merenstein, Jeremy Ward, David Allen}

Author affiliations: Boston University School of Medicine, Boston, Massachusetts, USA (C. Merenstein); Middlebury College,

Middlebury, Vermont, USA (J. Ward, D. Allen)

DOI: https://doi.org/10.3201/eid2605.191135

An unexpected Diplorickettsia species closely related to the tickborne pathogen $D$. massieliensis was found in the microbiome of an Ixodes scapularis tick in Vermont, USA. This evidence of Diplorickettsia in North American ticks suggests a need for disease surveillance using molecular screening of ticks and serologic studies of humans.

T he blacklegged tick, Ixodes scapularis, is a generalist arthropod ectoparasite that serves as a vector for an array of common human pathogens; novel disease-causing microbes have been discovered consistently in the tick for the past several decades. Tickborne bacterial infections causing illnesses such as anaplasmosis, Borrelia miyamotoi disease, and ehrlichiosis have all emerged in the United States in recent years (1-3), and it has been estimated that as many as half of all tickborne illnesses are caused by unknown pathogens (4).
We used 16S rRNA sequencing to survey for bacterial pathogens in I. scapularis ticks in western Vermont, USA. We collected ticks by drag sampling along $100 \mathrm{~m}$ transects using a $1 \mathrm{~m}^{2}$ square of white denim. We collected ticks from 6 deciduous forest sites in Addison and Chittenden counties, Vermont (Appendix Table 1, https://wwwnc.cdc.gov/EID/ article/26/5/19-1135-App1.pdf), during May-July 2015. We extracted DNA from ticks using a phenolchloroform extraction (5). In total, we extracted DNA from 97 ticks, 20 of which we selected based on DNA quality and quantity for $16 \mathrm{~S}$ rDNA sequencing at Hudson Alpha Genomic Services Laboratory (Huntsville, AL, USA). We PCR amplified the V3 and V4 regions of the $16 \mathrm{~S}$ rRNA gene from these ticks and 1 blank using $341 \mathrm{~F}$ and $875 \mathrm{R}$ primers (6) and sequenced them on a MiSeq platform (Illumina, https://www. illumina.com), yielding a total of 15,302,568 reads. We used the DADA2 R package to identify amplicon sequence variants (ASVs) and assign taxonomy (7) (Appendix Figure 1). We used default settings in the DADA2 pipeline; however, we estimated error rates using the first 10 billion base pairs.

In a single adult male tick, an ASV assigned to the genus Diplorickettsia comprised $82 \%$ of the microbiome sequencing reads. The genus Diplorickettsia was originally defined by the species $D$. massiliensis, discovered in I. ricinus ticks in Europe (8). The ASV we identified shared 425 of $427 \mathrm{nt}$ in the sequenced V3$\mathrm{V} 4$ region of the $16 \mathrm{~S}$ rRNA gene with the reference

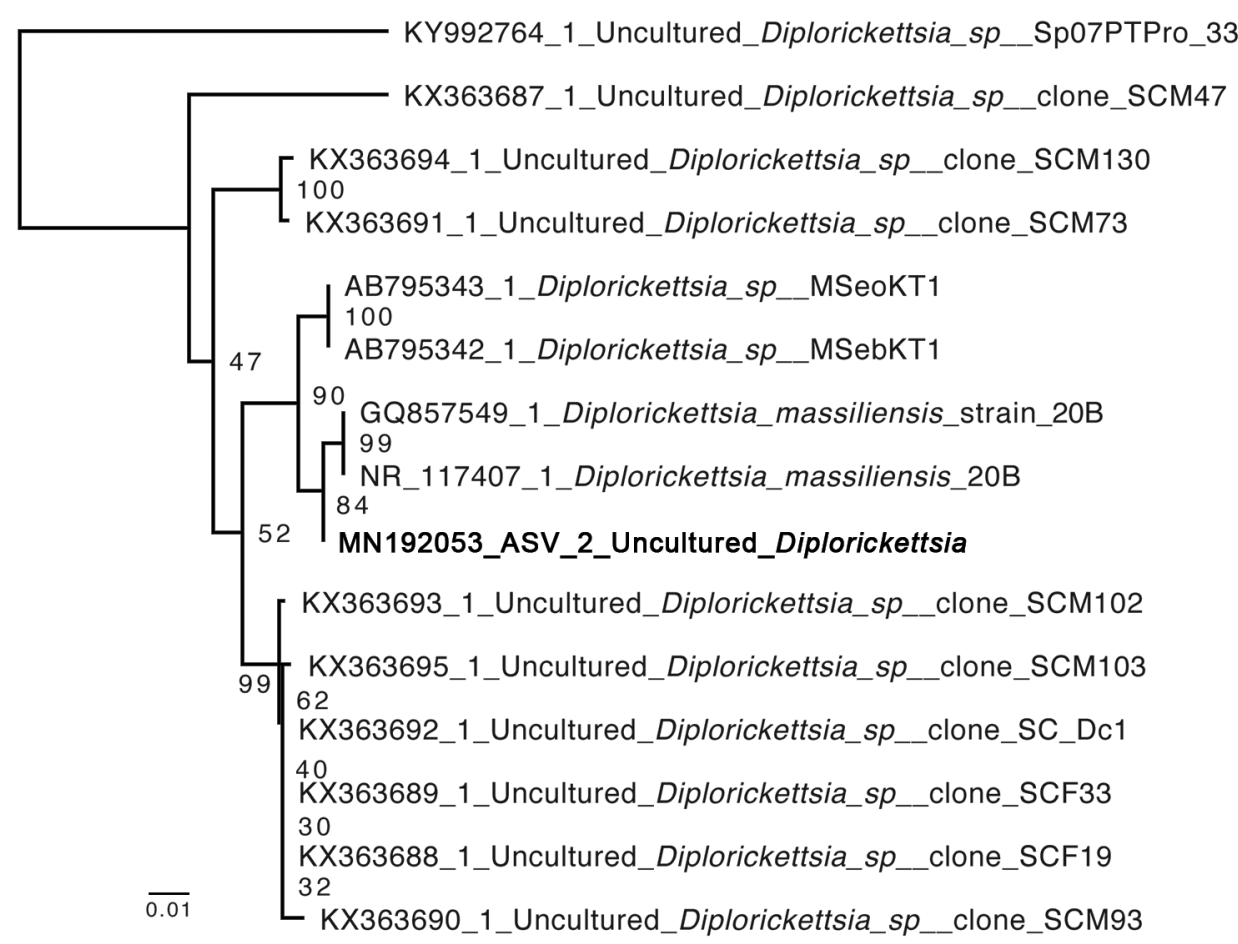

Figure. Neighbor-joining phylogenetic tree of a MAFFT alignment (https://mafft.cbrc.jp/ alignment/server) of the V3-V4 region of the Diplorickettsia $16 \mathrm{~S}$ rRNA gene, including the novel amplicon sequence variant identified in Vermont, USA (bold). A total of 427 bases were aligned and 363 conserved sites were used for neighbor-joining phylogeny, with 100 bootstrap iterations. The $341 \mathrm{~F}$ and $875 \mathrm{R}$ primers were used to amplify these regions (6). Default alignment parameters were used for alignment and generation of phylogenetic tree. Numbers at nodes indicate bootstrap values after 1,000 bootstrapping iterations. GenBank accession numbers are indicated. Scale bar represents average number of substitutions per site. 
sequence of $D$. massiliensis strain 20B (GenBank accession no. NR_117407.1) and was more closely related to this strain than any other previously sequenced Diplorickettsia in the National Center for Biotechnology Information (NCBI) nucleotide database (Figure). This tick was collected at the Sunny Hollow Colchester site (coordinates $44.518353^{\circ},-73.17112^{\circ}$ ) (Appendix Table 1).

D. massiliensis has been identified as a possible human pathogen; of patients in a hospital in France, 3 were found seropositive, and 1 found positive by quantitative PCR for the D. massiliensis rpoB gene (9). Our findings represent evidence of a Diplorickettsia bacteria in ticks in North America.

To confirm the presence of Diplorickittsia in the positive tick, we designed PCR primers to regions of the D. massiliensis parC and fts Y genes (Appendix Table 2) using primer-BLAST (https://www.ncbi. nlm.nih.gov/tools/primer-blast). We aligned primers against the NCBI nr database to ensure specific binding to Diplorickettsia. We also used DNA from a Diplorickettsia-negative tick (as determined by $16 \mathrm{~S}$ sequencing) as PCR template to serve as a negative control. Successful amplification of regions of both genes confirmed the presence of Diplorickettsia DNA in the positive tick (Appendix Figure 2).

We Sanger sequenced amplicons from these PCR tests. We combined forward and reverse reads and trimmed them using the PEAR utility (https:/ / cme.h-its.org/exelixis/web/software/pear). Each sequence showed high identity with previously sequenced $D$. massiliensis reference sequences via gapped alignment $(247 / 252$ bp parC, 298/310 bp fts Y). These results further suggest a close relationship between Diplorickettsia species we identified and D. massiliensis, but a lack of reference sequences for these genes from other species of Diplorickettsia makes it impossible to definitively assign this uncultured specimen to a particular species.

The high sequence similarity between the Diplorickettsia we identified and the previously identified pathogenic variety suggests the need for further study of the pathogenicity of this variant. Many genera of tickborne bacteria contain both pathogenic and nonpathogenic strains, and genetic similarity alone cannot confirm pathogenicity. Future work is needed to isolate this strain of Diplorickettsia and determine its ability to infect mammalian hosts and its transmissibility via tick bite. Experiments to test its ability to induce febrile illness in mammals would also help determine if Diplorickettsia spp. could cause a clinically significant infection in humans. Furthermore, serologic studies of patients with suspected tickborne diseases in the area surrounding the collection site are necessary to determine if this bacterium has infected persons in Vermont.

In addition, our findings suggest the need for further study of the prevalence of Diplorickettsia in North America ticks. We have developed PCR primers (Appendix Table 2) to facilitate future study of this bacterium and have demonstrated via sequencing that these primers accurately amplify their target Diplorickettsia genes. We have deposited the partial Diplorickettsia $16 \mathrm{~S}$ rRNA, fts Y, and parC genes sequenced in this study into the NCBI GenBank database (accession nos. MN192053, MN640996, MN640997). The raw sequencing reads are available through the NCBI sequence read archive (accession no. PRJNA557440).

Research reported in this publication was supported by an Institutional Development Award (IDeA) from the National Institute of General Medical Sciences of the National Institutes of Health under grant no. P20GM103449. Additional funding was provided by the Middlebury College Biology Department and the Albert D. Mead Professorship at Middlebury College.

\section{About the Author}

Mr. Merenstein is a computational analyst at Boston University School of Medicine, Boston, Massachusetts. His primary interests include microbial ecology and host-associated microbes.

\section{References}

1. Buller RS, Arens M, Hmiel SP, Paddock CD, Sumner JW, Rikhisa Y, et al. Ehrlichia ewingii, a newly recognized agent of human ehrlichiosis. N Engl J Med. 1999;341:148-55. https:// doi.org/10.1056/NEJM199907153410303

2. Dumler JS, Choi K-S, Garcia-Garcia JC, Barat NS, Scorpio DG, Garyu JW, et al. Human granulocytic anaplasmosis and Anaplasma phagocytophilum. Emerg Infect Dis. 2005;11:182834. https:/ / doi.org/10.3201/eid1112.050898

3. Telford SR III, Goethert HK, Molloy PJ, Berardi VP, Chowdri HR, Gugliotta JL, et al. Borrelia miyamotoi disease: neither Lyme disease nor relapsing fever. Clin Lab Med. 2015;35:867-82. https:/ / doi.org/10.1016/ j.cll.2015.08.002

4. Feder HM Jr, Johnson BJB, O'Connell S, Shapiro ED, Steere AC, Wormser GP; Ad Hoc International Lyme Disease Group. A critical appraisal of "chronic Lyme disease." N Engl J Med. 2007;357:1422-30. https:/ / doi.org/10.1056/ NEJMra072023

5. Van Treuren W, Ponnusamy L, Brinkerhoff RJ, Gonzalez A, Parobek CM, Juliano JJ, et al. Variation in the microbiota of Ixodes ticks with regard to geography, species, and sex. Appl Environ Microbiol. 2015;81:6200-9. https:// doi.org/10.1128/ AEM.01562-15

6. Klindworth A, Pruesse E, Schweer T, Peplies J, Quast C, Horn M, et al. Evaluation of general 16S ribosomal RNA gene PCR primers for classical and next-generation 
sequencing-based diversity studies. Nucleic Acids Res. 2013;41:e1. https://doi.org/10.1093/nar/gks808

7. Callahan BJ, McMurdie PJ, Rosen MJ, Han AW, Johnson AJA, Holmes SP. DADA2: high-resolution sample inference from Illumina amplicon data. Nat Methods. 2016;13:581-3. https://doi.org/10.1038/nmeth.3869

8. Mediannikov O, Sekeyová Z, Birg M-L, Raoult D. A novel obligate intracellular gamma-proteobacterium associated with ixodid ticks, Diplorickettsia massiliensis, gen. nov., sp. nov. PLoS One. 2010;5:e11478. https://doi.org/10.1371/ journal.pone.0011478

9. Subramanian G, Mediannikov O, Angelakis E, Socolovschi C, Kaplanski G, Martzolff L, et al. Diplorickettsia massiliensis as a human pathogen. Eur J Clin Microbiol Infect Dis. 2012;31: 365-9. https://doi.org/10.1007/s10096-011-1318-7

Address for correspondence: David Allen, Department of Biology, Middlebury College, McCardell Bicentennial Hall 372, Middlebury, VT 05753, USA; email: dallen@middlebury.edu

\section{Case of Babesia crassa-Like Infection, Slovenia, 2014}

\author{
Katja Strasek-Smrdel, Misa Korva, Emil Pal, \\ Mojca Rajter, Miha Skvarc, Tatjana Avsic-Zupanc
}

\begin{abstract}
Author affiliations: Institute of Microbiology and Immunology, Faculty of Medicine, University of Slovenia, Ljubljana, Slovenia (K. Strasek-Smrdel, M. Korva, M. Skvarc, T. Avsic-Zupanc); Murska Sobota General Hospital, Rakican, Slovenia (E. Pal); University Medical Centre Ljubljana, Ljubljana (M. Rajter)
\end{abstract}

DOI: https://doi.org/10.3201/eid2605.191201

We report a case of Babesia crassa-like infection in an asplenic patient in Slovenia in 2014. We diagnosed the infection using microscopy, 18S rRNA sequencing, and serology and monitored parasitemia using digital PCR. With its increasing occurrence, babesiosis should be included in differential diagnoses for immunocompromised patients displaying fever.

B abesia infections occur worldwide and cause disease mainly in animals, but disease occurs occasionally in humans. Infections in humans are mostly attributable to B. microti, B. duncani, and Babesia sp. MO1 in North America; B. divergens, B. venatorum, and B. microti in Europe; and B. venatorum, B. crassalike parasite, B. microti, Babesia sp. XXB/HangZhou, and Babesia sp. KO-1 in Asia $(1,2)$. Transmission occurs predominantly through tick bites, but humans have acquired infections via contaminated blood products and through the transplacental and perinatal routes (1). Most patients with Babesia infections in Europe were reported to be asplenic or immunocompromised. Typical clinical signs and symptoms include fever (up to $40^{\circ} \mathrm{C}$ ), parasitemia $(20 \%-80 \%)$, severe anemia, muscle weakness, fatigue, and lateonset jaundice with dark urine, and sometimes complications develop. Long-term clinical follow-up that includes blood smear examination and PCR analysis is necessary because relapse and persistence of parasitemia can occur in spite of treatment. The application of novel molecular methods has revealed that the host range of many Babesia species is less restricted than previously thought. New species or animal pathogens are increasingly being discovered as causing Babesia infections in humans. We report a B. crassa-like infection in a patient in Slovenia in 2014.

In 2014, a 55-year-old woman, living on the outskirts of Murska Sobota, Slovenia, sought medical treatment for a 6-day history of intermittent fever up to $39^{\circ} \mathrm{C}$, myalgia, headache, poor appetite concomitant with weight loss, fatigue, sweating, and dark urine. She previously had a splenectomy and partial pancreatectomy 5 years previous because of cystic adenoma and adrenal incidentaloma without hormonal activity. She reported no history of travel, tick bite, animal contact, or blood transfusions.

Her blood pressure was 115/70 $\mathrm{mm} \mathrm{Hg}$, heart rate 83 beats/min, and body temperature $36.6^{\circ} \mathrm{C}$, and a physical examination indicated no significant clinical findings. The first basic blood analysis revealed thrombocytopenia (platelets $85 \times 10^{9} / \mathrm{L}$ ). A differential blood analysis indicated that the concentration of large unstained cells was elevated $(0.41$ $\times 10^{9} / \mathrm{L}$, reference range $0-0.4 \times 10^{6} / \mathrm{L}$ ). Biochemical laboratory testing showed mild fluctuations in liver functioning: aspartate aminotransferase 1.22 (reference range 0.17-0.51) $\mu \mathrm{kat} / \mathrm{L}$, alanine aminotransferase 1.13 (reference range 0.17-0.68) $\mu \mathrm{kat} / \mathrm{L}$, $\gamma$-glutamyltransferase 1.08 (reference range 0.03-0.51 $\mu \mathrm{kat} / \mathrm{L}$ ) $\mu \mathrm{kat} / \mathrm{L}$, and alkaline phosphatase 1.88 (reference range 0.5-2.0) $\mu \mathrm{kat} / \mathrm{L}$. C-reactive protein was $51 \mathrm{mg} / \mathrm{L}$ (150 [reference range 0.76-28.5] nmol/L), and mild erythrocyturia was present. Giemsa-stained blood smears showed unusual inclusions in erythrocytes, Howell-Jolly bodies, mild anisocytosis, some atypical lymphocytes, and some large thrombocytes. We observed many ring forms and some paired piriform shapes of Babesia spp. in blood smears (Figure), and parasitemia was 1\% (Appendix Table, 